\title{
The Embedding of Spacetime into Cauchy Developments
}

\author{
F. Dahia ${ }^{1}$ and C. Romero ${ }^{2}$ \\ ${ }^{1}$ Departamento de Física, UFCG, 58109-970, Campina Grande-PB, Brazil and \\ ${ }^{2}$ Departamento de Física, UFPB, \\ Cx. 5008, 58051-970, João Pessoa -PB, Brazil
}

(Received on 15 October, 2005)

\begin{abstract}
Using the Campbell-Magaard theorem we show that any analytic spacetime can be locally embedded into the Cauchy development of legitimate initial data for the five dimensional vacuum Einstein equations. The embedding presents the domain of dependence property and the Cauchy stability with respect to those initial data.
\end{abstract}

\section{INTRODUCTION}

Nowadays the subject of extra dimensions has attracted the attention of physics motivated by the so-called braneworld scenarios [1, 2]. According to these models the ordinary spacetime (the observed spacetime associated to the phenomena whose energy is below the scale of $\mathrm{TeV}$ ) is a fourdimensional submanifold embedded in an ambient space with higher dimension.

It happens that the embedding of a space into another can not be made freely. The embedding demands certain geometric compatibility between the spaces. Hence it is natural to ask whether these models impose some constraints on the geometry of the ordinary spacetime we observe. Does every fourdimensional spacetime can be obtained in these scenarios?

Of course this issue only can be properly addressed in the context of embedding theorems. There are well known general results which guarantee that any manifold can be isometrically embedded in some flat space of sufficient high dimensions. The dimension of the ambient space depends on the type of the embedding considered. For example, if the embedding is local and analytic then $n(n+1) / 2$ is the maximum number of dimensions we would need to embed any $n$ dimensional manifold [3]. Considering a $C^{\infty}$ local embedding this number increases to $n(n+3) / 2$ [4].

Here we are concerned with models which propose ambient spaces with five dimensions, as the Randall-Sundrum (RS) type models [2] and the so-called non-compactified KaluzaKlein models (NKK)[5]. In these cases, the ambient space has only one extra dimension. However its geometry is not prescribed a priori. Instead it is admitted that the ambient space satisfies field equations: Einstein vacuum equations (NKKmodels) or Einstein equation with the cosmological constant (RS type models).

The Campbell-Magaard theorem and its variants are of special relevance for these models[7]. It can be stated in following way: Given any $n$-dimensional analytical space $(M, g)$, where $g$ stands for the metric, for any point $p \in M$ there exists a vacuum solution of Einstein equations in $(n+1)$-dimensions into which a neighborhood of $p$ in $M$ can be embedded.

However showing the existence of the embedding is not enough for physical purposes. It is important to ensure that the embedding possesses the desirable physical properties of stability and domain of dependence. The CM theorem is not appropriate to address these issues since it is based on Cauchy-
Kowalewski theorem which demands the analyticity of the functions [8].

In this paper we shall reconsider the embedding from another perspective. We have found that for any given analytic spacetime, there correspond initial data sets, legitimate for the problem of Cauchy in General Relativity, in whose Cauchy development for five-dimensional vacuum Einstein equations the spacetime can be locally embedded [9]. In this sense, the embedding of the spacetime might be understood as the result of the physical evolution of the initial data. This formulation have some advantages in comparison with the original $\mathrm{CM}$ theorem, since we can establish the stability and domain of dependence properties of the embedding relative to the initial data. For details, the reader is referred to [9].

\section{DYNAMICAL APPROACH}

The usual procedure adopted to solve the embedding problem concerning the CM theorem is similar to an initial value problem in General Relativity. Schematically we can say that the spacetime $(M, g)$ is taken as part of initial data and it is propagated by the dynamical part of the vacuum Einstein equations along the extra dimension in order to generate the five-dimensional space $(\widetilde{M}, \widetilde{g})$ into which the spacetime is embedded. However this propagation is not a real physical evolution since the direction of evolution is spacelike. Hence, for example, there is no reason why we should expect a causal connection between different slices of the ambient space.

Nevertheless we can show that there exist a 5D neighborhood $\widetilde{O}$ of $p$ in $\widetilde{M}$ and a function $\phi$ such that its gradient $\nabla \phi$ is timelike everywhere in $\widetilde{O}$. Therefore the inverse images of $\phi$ are acausal spacelike hypersurfaces of $\widetilde{O}$. Let us consider $\Sigma$ the hypersurface that contains the point $p$. Since $\nabla \phi$ is timelike then the induced metric in $\Sigma$ by the embedding of this hypersurface in $(\widetilde{O}, \widetilde{g})$ is positive definite. Consider now the extrinsic curvature $K$ of $\Sigma$ relative to $(\widetilde{O}, \widetilde{g})$. By construction, $\Sigma$ is a hypersurface of $5 \mathrm{D}$ vacuum space, then, it is clear that $h$ e $K$ satisfy the vacuum constraint in $\Sigma$. Therefore, the triple $(h, K, \Sigma)$ constitutes a legitimate initial data set for the Cauchy problem in General Relativity[10].

Consider now $D(\Sigma)$, the domain of dependence of $\Sigma$ relative to $(\widetilde{O}, \widetilde{g})$. It can be shown (see [11], page 425 ) that since 


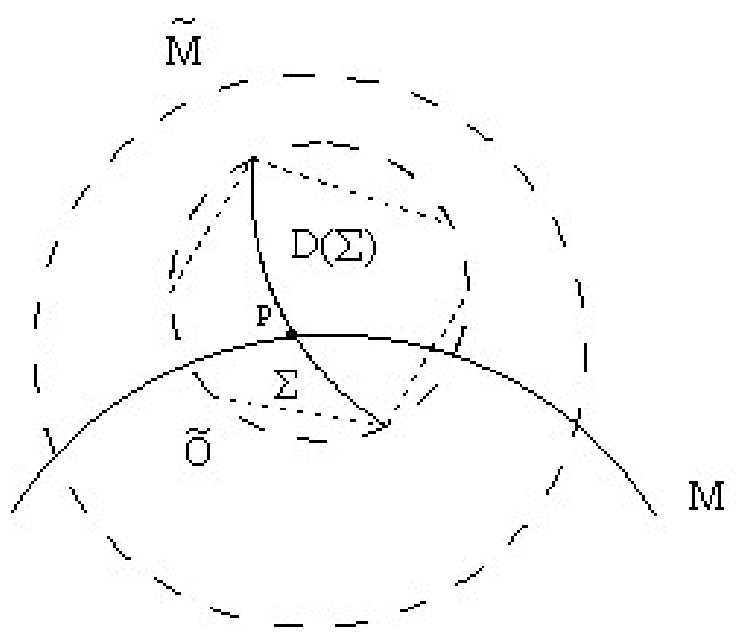

FIG. 1: Sketch of the local embedding of spacetime $M$ into the Cauchy development $D(\Sigma)$ of initial data given in an acausal spacelike four dimensional hypersurface $\Sigma$.

$\Sigma$ is an acausal hypersurface of $\widetilde{O}$, then $D(\Sigma)$ is open in $\widetilde{O}$. Of course $D(\Sigma)$ is a non-empty set, since $\Sigma \in D(\Sigma)$. The domain of dependence $D(\Sigma)$ is an open subset of $\widetilde{M}$. Endowed with the vacuum metric $\widetilde{g}$, it represents a five-dimensional manifold $(D(\Sigma), \widetilde{g})$ which is a solution of the vacuum Einstein equations. This means that $(D(\Sigma), \widetilde{g})$ is a Cauchy development for the Einstein vacuum equations of the initial data $(h, K, \Sigma)$, that is, it corresponds to the manifold which arises from physical evolution of the initial data.

Now we shall show that there is a region of the spacetime $M$ which is embedded in $D(\Sigma)$. We know that $M$ and $D(\Sigma)$ are open and both contain the point $p$. Then $M \cap D(\Sigma)$ is a non- empty open set which is a neighborhood of $p$ in $M$ contained in $D(\Sigma)$. Therefore $(M \cap D(\Sigma), g)$ is embedded in $(D(\Sigma), \widetilde{g})$, i.e., in a Cauchy development of $(h, K, \Sigma)$. In other words the dynamical evolution of the initial data $(h, K, \Sigma)$ generates a five-dimensional vacuum space into which the spacetime is locally embedded. In this sense, we can say that this local embedding is dynamically generated by the physical propagation of those initial data (see figure).

This perspective have some advantages because it allow us to establish the domain of dependence property and the stability of the embedding relative to the initial data $(h, K, \Sigma)$. This is a direct consequence of the fact that the spacetime is embedded into the Cauchy development of $(h, K, \Sigma)$ which, as it is well known[10], exhibit these properties.

Indeed if we consider another point $q \in M$ in the future domain of dependence $D^{+}(\Sigma)$ it follows that the $g(q)$, the value of the metric at $q$, depends only on $J^{-}(q) \cap \Sigma$, that is, the initial data in the causal past of $q$. This is the domain of dependence property and it guarantees that any perturbation outside $J^{-}(q) \cap \Sigma$ does not affect the spacetime at $q$ since the embedding is causal connected to the initial data and the signals travel with a finite speed.

Another very interesting property is the Cauchy stability. It means that dependence of the induced metric in $M$ is continuous with respect the initial data. Roughly speaking it express the idea that new initial values $h^{\prime}$ and $K^{\prime}$ sufficiently close to the $h$ and $K$, in respect to the Sobolev norm (for details, see [9]), give rise to a new metric which will induce a metric in $M$ which is Lorentzian and close to the original spacetime metric $g$.

\section{Acknowledgements}

The authors thank CNPq-Fapesq (Pronex) for financial support.
[1] I. Antoniadis, N. Arkani-Hamed, S. Dimopoulos, and G. Dvali, Phys. Lett. B 436, 257 (1998).

[2] L. Randall and R. Sundrum, Phys. Rev. Lett. 83, 4690 (1999).

[3] M. Janet, Ann. Soc. Polon. Math. 5, 38 (1926). E. Cartan, Ann. Soc. Polon. Math. 6, 1 (1927)

[4] R. Greene, Memoirs Amer. Math. Soc. 97, 1 (1970)

[5] J. M. Overduin, P. S. Wesson, Phys. Rep. 283, 303 (1997).

[6] J. Campbell, A Course of Differential Geometry (Oxford: Claredon, 1926). L. Magaard, (PhD Thesis, Kiel, 1963). C. Romero, R. Tavakol, and R. Zalaletdinov, Gen. Rel. Grav. 28, 365 (1996).

[7] E. Anderson and J. Lidsey, Class. Quantum Grav. 18, 4831 (2001). F. Dahia and C. Romero, J. Math. Phys., 43, 11, 5804, (2002). F. Dahia and C. Romero, J. Math. Phys. 43, 6, 3097
(2002). E. Anderson, F. Dahia, J. Lidsey, and C. Romero, J. Math. Phys.44, 5108 (2003). S. Seahra and P. Wesson, Class. Quantum Grav. 20, 1321 (2003). N. Katzourakis, math$\mathrm{ph} / 0407067$.

[8] E. Anderson, gr-qc/0409122 E. Anderson and R. Tavakol, grqc/0309063.

[9] F. Dahia and C. Romero, Class. Quant. Grav. 22, 5005, (2005).(gr-qc/0503103).

[10] S. Hawking and G. Ellis, The large scale structure of spacetime, (Cambridge Univ. Press, 1973).

[11] B. O'Neill, Semi-Riemannian Geometry, (Academic Press, 1983). 